\title{
Selective photoionization of palladium isotopes using a two-step excitation scheme
}

\author{
Clayton R. Locke ${ }^{1} \cdot$ Tohru Kobayashi $^{1} \cdot$ Takashige Fujiwara $^{1} \cdot$ Katsumi Midorikawa $^{1}$
}

Received: 5 June 2017 / Accepted: 22 August 2017 / Published online: 29 August 2017

(c) The Author(s) 2017. This article is an open access publication

\begin{abstract}
We present a novel two-step even-odd mass isotope selective excitation and ionization scheme, potentially applicable in resource recycling and management of palladium occurring in high-level nuclear waste. In contrast to the conventional three-step selective ionization process, the two-step scheme utilizes transition selection rules to an autoionizing Rydberg state, rather than to an intermediate state, resulting in an increase in efficiency of over an order of magnitude while retaining excellent selectivity of $>99.7 \%$. The reduction in the number of excitation lasers required allows several technical simplifications and reduces costs should the process be developed for large-scale resource recycling operations.
\end{abstract}

\section{Introduction}

Palladium is a valuable metal in industry, and occurs as one of the fission products in nuclear power plant radioactive waste in quantities comparable to those available from natural reserves [1]. However, it is unable to be utilized due to the co-presence of the long lived ( 6.5 million years) isotope ${ }^{107} \mathrm{Pd}$, comprising $17 \%$ of the total amount of palladium in the waste. It is imperative, in both economic and environmental senses, to establish operations to selectively remove this radioactive isotope [2].

Electronic supplementary material The online version of this article (doi:10.1007/s00340-017-6818-4) contains supplementary material, which is available to authorized users.

Clayton R. Locke

clayton.locke@riken.jp

1 RIKEN Center for Advanced Photonics, 2-1 Hirosawa, Wako, Saitama 351-0198, Japan
Selective ionization using a narrow-linewidth laser to excite only specific isotopes, such as is used in the selective ionization of ${ }^{235} U$ [3], is not applicable for palladium as isotope frequency shifts are smaller than the Dopplerbroadened absorption linewidths [4]. Instead, we base our work on a technique first proposed by Balling and Wright [5] wherein optical selection rules forbid excitation of evenmass number isotopes, but allow excitation of odd-mass number isotopes $\left({ }^{105} \mathrm{Pd}\right.$ and ${ }^{107} \mathrm{Pd}$ ) having non-zero nuclear spin. This is achieved using either two circularly polarized [6] (or orthogonal linearly polarized [7]) excitation lasers interacting with a stream of vaporized palladium, followed by a third ionizing laser. Ions are then separated from the vapor by an electric field, and non-radioactive isotopes remaining in the stream can be recycled, and odd-mass number isotopes (including radioactive ${ }^{107} \mathrm{Pd}$ ) can be processed by means such as nuclear transmutation [8].

This optical transition selection rule lends itself well to industrial scale-up, having notable advantages when compared to competing methods. Specifically, commercial high power excitation lasers can be used, without the need for narrow spectral linewidth nor long-term frequency stability, as is required for Atomic Vapor Laser Isotope Separation (AVLIS) [9]. Secondly, yield production is not limited, as may be the case when scaling up resonant ionization mass spectrometry RIMS [10] processes. This later limitation we have directly observed when a moderately high number of ions (of order $10^{11}$ ) is produced in the interaction region and ion-ion Coulomb repulsive forces result in significant degradation of mass resolution (by either accelerating electric or magnetic fields), to the point where isotope resolution by a narrow slit is impossible.

In previous work, we demonstrated the overall yield can be improved by tuning the third laser to autoionizing Rydberg states [11], where we used the $J_{\mathrm{c}} K$ coupling scheme 
[12] for assignment of energy levels, which is appropriate for Pd I. The ionization yield was then further improved using an alternative excitation pathway [13] of intermediate states having ${ }^{2} \mathrm{D}_{3 / 2}$ core.

We present here a significantly novel scheme, whereby transition selection rules to the autoionizing Rydberg states are exploited, rather than those to an intermediate state, as illustrated in Fig. 1. In contrast to previously reported schemes via a $4 d[14,15], 5 d$ [11], or $6 s$ [13] intermediate state before the Rydberg state, this two-step selective ionization scheme is via a $5 p$ intermediate state.

Previous studies of two-photon excitation through a $5 \mathrm{p}$ state $[10,16]$ have resolved high-lying autoionizing $n s$ and $n$ d Rydberg states, although a comprehensive report (including relative intensities and definitive identification) has not to-date been carried out. This information is vital in identifying the autoionizing state having the best combination of ionization efficiency and even-odd mass isotope selectivity. We show the two-step technique exhibits excellent selectivity of over $99.7 \%$ in ionizing only odd-mass number isotopes.

\section{Experiment}

The apparatus used has been described in detail previously [7], and is only briefly summarized here, as shown schematically in Fig. 2. An electron bombardment source was used to heat a crucible of palladium to approximately $2000 \mathrm{~K}$

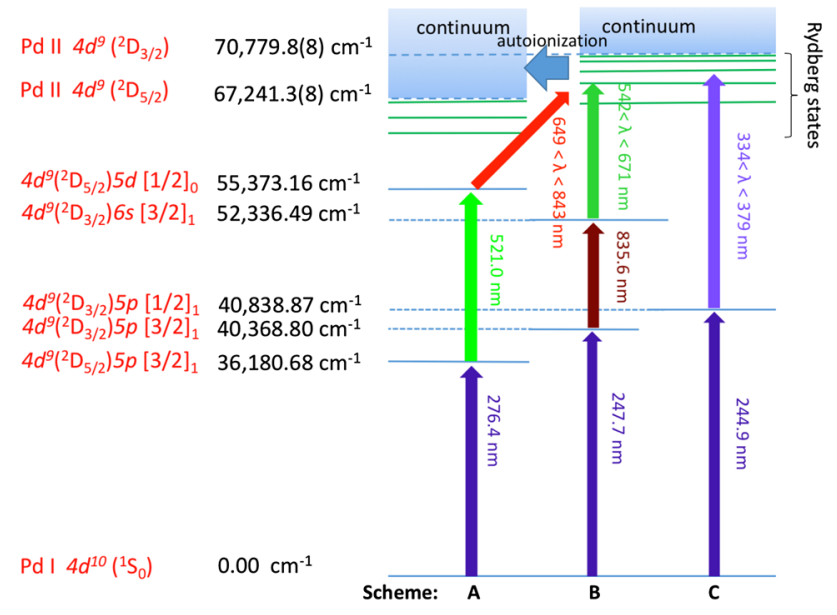

Fig. 1 Even-odd mass isotope selective excitation schemes: $A$ the conventional three-laser scheme using ${ }^{2} \mathrm{D}_{5 / 2}$ core intermediate states with two lasers having circular (or linear orthogonal) polarized light; $B$ three-laser scheme with isotope selectivity obtained via parallel polarized light using a ${ }^{2} \mathrm{D}_{3 / 2}$ core intermediate states; $C$ the two-step selective excitation scheme proposed in this work using either parallel or perpendicular polarization using a ${ }^{2} \mathrm{D}_{3 / 2}$ core intermediate state. Energies and level designations are from NIST Atomic Spectra Database [17]

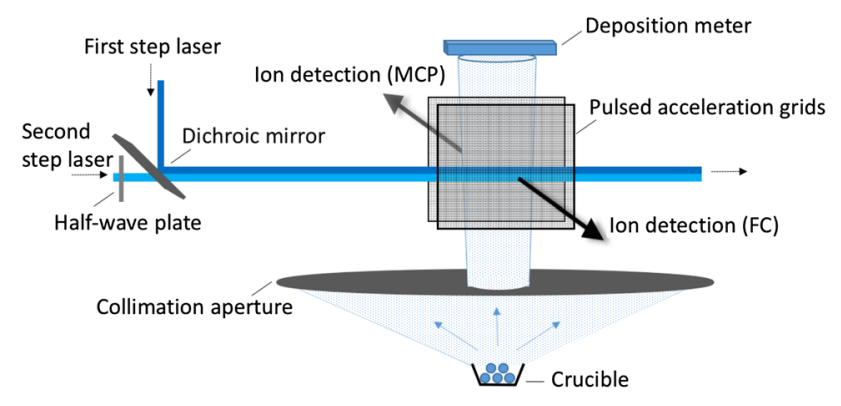

Fig. 2 Schematic showing the introduction of two co-propagating lasers to interact with palladium vapor. The voltage on grids (activated approximately $2 \mu \mathrm{s}$ after the interaction) created an electric field in the interaction region that was able to be reversed, either to accelerate ions towards a Faraday Cup (FC) detector or Micro Channel Plate (MCP)

to produce vaporized palladium, collimated by a $20 \mathrm{~mm}$ diameter aperture which is charged with a positive potential to deflect ions produced in the vaporization away from the interaction region. For laboratory safety, preliminary experiments used palladium samples with only naturally occurring isotopes (i.e., no ${ }^{107} \mathrm{Pd}$ is present); however, as the selectivity scheme is expected to behave identically for both ${ }^{105} \mathrm{Pd}$ and ${ }^{107} \mathrm{Pd}$ (both have nuclear spin of $I=5 / 2$ ), we use the abundance of ${ }^{105} \mathrm{Pd}$ as a gauge of selectivity performance. The relative number of atoms present in the interaction region was measured using a deposition meter (Inficon STM-2 Thin Film Rate/Thickness Monitor) located above the interaction region.

Vaporized palladium and the lasers intersected in a fieldfree volume, and ions produced from the interaction were accelerated by a voltage gradient (activated approximately $2 \mu \mathrm{s}$ after the interaction) from grids in Wiley-McLaren configuration [18]. Depending on the polarity of the acceleration grids, ions were directed either through a 1-meter time-of-flight tube and detected by a 2-stack microchannel plate (MCP), or to a Faraday Cup (FC) detector. The massspectrometer had mass resolution $(\mathrm{m} / \Delta \mathrm{m})$ of 600 , and was used to measure the degree of selectivity of odd-to-even mass number isotopes. The FC was used to determine the overall yield, both in terms of estimating the number of ions striking the detector shot-to-shot from the electrical signal, and also for long-term deposition (for later weighing and chemical analysis) upon a gold-plated slide forming the back plate of the FC. The FC was located as close as possible to the interaction region and had a large area, and, unlike the $\mathrm{MCP}$, was able to capture all ions and was not saturated at high ion production rates.

An excimer laser (Lambda Physik Compex 103) pumps two dye lasers (Lambda Physik FL3002), producing synchronous pulses of width of $20 \mathrm{~ns}$ and repetition rate $10 \mathrm{~Hz}$. The first dye laser (using Coumarin 102 dye) is frequency 
doubled in a BBO crystal to produce the first excitation wavelength, which in the case of the two-step scheme is $489.730 \mathrm{~nm}$ and frequency doubled to $244.865 \mathrm{~nm}$, populating Pd atoms from the ground state to $4 d^{9}\left({ }^{2} \mathrm{D}_{3 / 2}\right) 5 p[1 / 2]_{1}$. The second dye laser required two dyes (PTP and DMQ) to scan the range $334-379 \mathrm{~nm}$. The second laser beam was combined with the first laser beam co-linearly, using a dichroic mirror. Wavelengths of both first and second step lasers were recorded during the experiment using a wavemeter (High Finesse: WS5 UV-II) having an absolute accuracy of $3 \mathrm{pm}$ calibrated using an optogalvanic neon gas cell.

The lifetime of the first excited state is 5-7 ns [19]. If the two-laser pulses are not temporally synchronous, population loss from the intermediate state leads to loss in the final yield. To ensure correct timing, the beam path for the second laser was modified and pulse arrival time was monitored on a high speed oscilloscope. Temporal synchronization was also important to minimize time evolution of magnetic substates, which has a direct effect on isotope selectivity due to precession of the angular momentum around the magnetic field [20]. Therefore, minimal time delay between pulses will give maximum ionization and also maximum even-odd mass isotope selectivity.

The linear polarization of the second laser was controlled using a half-wave plate before the dichroic mirror to set it either perpendicular or parallel relative to the first basis-setting laser. If the two lasers have parallel polarization, selection rules $\left(\Delta m_{J}=0\right)$ forbid excitation through the pathway $J=0 \rightarrow 1 \rightarrow 1$ for even-mass number isotopes (having zero nuclear spin), yet is allowed for odd-mass number isotopes (having non-zero nuclear spin). Similarly, if the two lasers have perpendicular polarization $\left(\Delta m_{J}= \pm 1\right)$, excitation is forbidden through the pathway $J=0 \rightarrow 1 \rightarrow 0$ for evenmass number isotopes, yet is allowed for odd-mass number isotopes.

\section{Results and discussion}

For the two-laser scheme via the $4 d^{9}\left({ }^{2} \mathrm{D}_{3 / 2}\right) 5 p[1 / 2]_{1}$ intermediate state (C of Fig. 1), selection rules allow six autoionizing Rydberg series (one having $J=0$, three having $J=1$, and two having $J=2$ ). We note that even-odd mass isotope selective ionization is also possible via the $4 d^{9} 5 p[3 / 2]_{1}$ intermediate state (reached via a $247.7 \mathrm{~nm}$ first excitation laser). Transition rules for this intermediate state allows the aforementioned Rydberg series, and also an extra two Rydberg series having $J=2$ (which are not even-odd mass isotope selective). The relative intensities of $4 d^{9} 5 p[1 / 2]_{1}$ and $4 d^{9} 5 p[3 / 2]_{1}$ are 1100 and 1700 , respectively [17], and given that the first laser power must be low enough not to result in detectable two-photon ionization [7], the choice between these two intermediate states is arbitrary. For completeness, we identify the two extra Rydberg series accessible via the $4 d^{9} 5 p[3 / 2]_{1}$ intermediate state, but for the remainder of the analysis we use the $4 d^{9} 5 p[1 / 2]_{1}$ intermediate state.

With the first laser wavelength fixed, the wavelength of the second step laser was stepped over the entire range (for both parallel and perpendicular polarization) between the two ionic ground states Pd II $4 d^{9}\left({ }^{2} \mathrm{D}_{5 / 2}\right)$ and Pd II $4 d^{9}\left({ }^{2} \mathrm{D}_{3 / 2}\right)$ to determine the ionization efficiency and isotope selectivity of each autoionizing state. The signal from the MCP was recorded on a high speed digital oscilloscope at each wavelength step, showing the arrival time of each isotope. As shown in Fig. 3, when the second laser wavelength excites the atom to a Rydberg state, a significant increase in signal is observed due to auto-ionization. Additionally, we are able to distinguish which isotopes are ionized according to arrival time upon the MCP. The total signal at each wavelength step is summed to build up a map of the entire Rydberg series as shown in Fig. 4.

We deduce the total angular momenta $J$ of each state from observation of isotope selectivity. Following this, the energies of the Rydberg series were fitted to the extended Ritz formula [21, 22] where we used the mass-corrected Rydberg constant of Pd I $\left(109,736.75 \mathrm{~cm}^{-1}\right)$. Ionization potential of Pd II $4 d^{9}\left({ }^{2} \mathrm{D}_{3 / 2}\right)$ and parameters $\delta_{0}$ and $\delta_{2}$ (the energy-dependent quantum defects that account

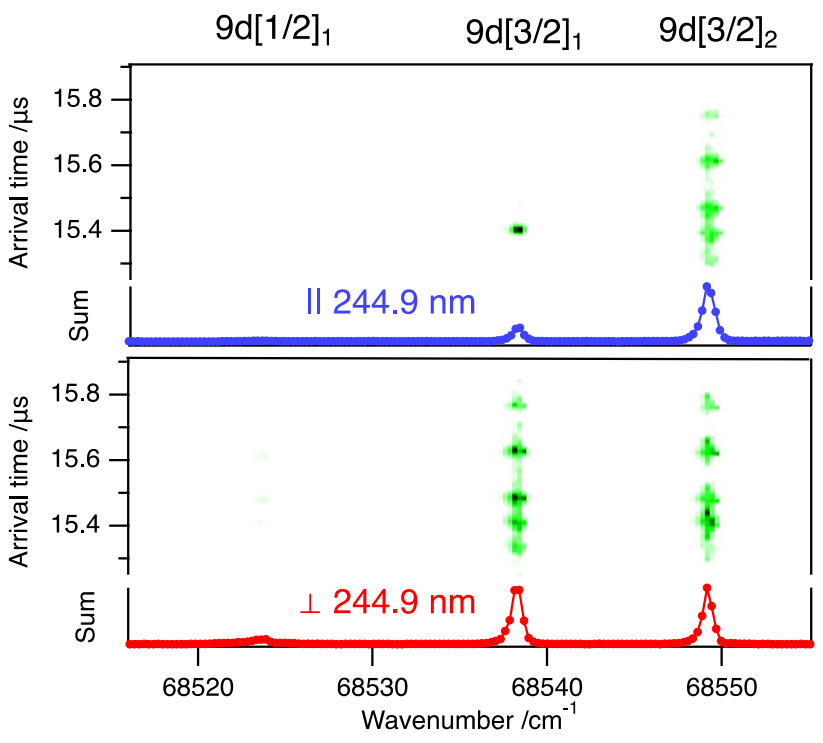

Fig. 3 Time-of-flight spectrum using the two-step excitation scheme with $244.9 \mathrm{~nm}$ intermediate state. Upper graph is when the relative polarization between laser beams is parallel $(\|)$, and only the odd mass isotope $\left({ }^{105} \mathrm{Pd}\right)$ is ionized and observed at $15.4 \mu \mathrm{s}$ for the $9 \mathrm{~d}[3 / 2]_{1}$ final state (and also weakly ionized for $9 \mathrm{~d}[1 / 2]_{1}$ ). The state $9 \mathrm{~d}[3 / 2]_{2}$ shows no even-odd mass isotope selective ionization and all isotopes are observed. Lower graph is when relative polarization between laser beams is perpendicular $(\perp)$ and all isotopes are observed for all final states 


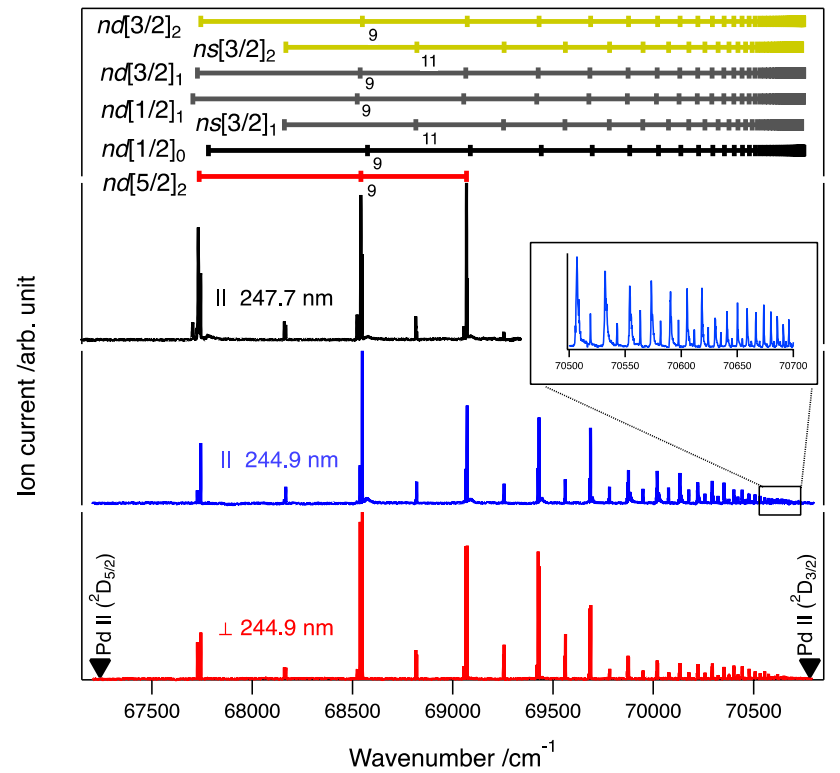

Fig. 4 Observed Rydberg spectrum using the two-step excitation scheme at perpendicular and parallel relative polarization. Top-most markers show positions of identified Rydberg series; || $247.7 \mathrm{~nm}$ : parallel polarization between the lasers using $247.7 \mathrm{~nm}$ intermediate state, where one extra Rydberg series $n \mathrm{~d}[5 / 2]_{2}$ is observed; \| $244.9 \mathrm{~nm}$ : parallel polarization between the lasers using $244.9 \mathrm{~nm}$ intermediate state; $\perp 244.9 \mathrm{~nm}$ : perpendicular polarization between the lasers using $244.9 \mathrm{~nm}$ intermediate state. Downward triangle ionic ground states of $\mathrm{Pd} \mathrm{II} 4 \mathrm{~d}^{9}\left({ }^{2} \mathrm{D}_{3 / 2}\right)$ and $\mathrm{Pd} \mathrm{II} 4 \mathrm{~d}^{9}\left({ }^{2} \mathrm{D}_{5 / 2}\right)$

for the shielding of the nucleus by the core electrons) were used as free fit parameters. The ionization potential of Pd II $\left(4 d^{9}{ }^{2} \mathrm{D}_{3 / 2}\right)$ was determined to be $70,780.67$ (23) $\mathrm{cm}^{-1}$, where the uncertainty was dominated by the linewidth of the lasers and absolute accuracy of the wavemeter $(0.002 \mathrm{~nm})$. This result is comparable with literature values of $70,779.8(8) \mathrm{cm}^{-1}$ [14], 70,780.9 (10) $\mathrm{cm}^{-1}[16], 70,780.38(8) \mathrm{cm}^{-1}[11]$, and 70,780.6(1) $\mathrm{cm}^{-1}$ [13]. Quantum defects resulting from the fit for each of the Rydberg series are listed in Table 1; residuals are less than $0.5 \mathrm{~cm}^{-1}$.

Table 1 Fitting coefficients ( \pm one standard deviation) to the extended Ritz formula; $\delta_{0}$ and $\delta_{2}$ are the energy-dependent quantum defects

\begin{tabular}{lll}
\hline & $\delta_{0}$ & $\delta_{2}$ \\
\hline $4 d^{9}\left({ }^{2} \mathrm{D}_{3 / 2}\right) n d[1 / 2]_{0}$ & $1.944(1)$ & $-0.27(4)$ \\
$4 d^{9}\left({ }^{2} \mathrm{D}_{3 / 2}\right) n s[3 / 2]_{1}$ & $3.522(1)$ & $-0.27(5)$ \\
$4 d^{9}\left({ }^{2} \mathrm{D}_{3 / 2}\right) n d[1 / 2]_{1}$ & $2.022(2)$ & $-0.26(10)$ \\
$4 d^{9}\left({ }^{2} \mathrm{D}_{3 / 2}\right) n d[3 / 2]_{1}$ & $1.999(1)$ & $-0.27(2)$ \\
$4 d^{9}\left({ }^{2} \mathrm{D}_{3 / 2}\right) n s[3 / 2]_{2}$ & $3.514(1)$ & $-0.24(5)$ \\
$4 d^{9}\left({ }^{2} \mathrm{D}_{3 / 2}\right) n d[3 / 2]_{2}$ & $1.984(1)$ & $-0.19(2)$ \\
$4 d^{9}\left({ }^{2} \mathrm{D}_{3 / 2}\right) n d[5 / 2]_{2}$ & $2.000(6)$ & $0.08(26)$ \\
\hline
\end{tabular}

We determined the principal quantum number of each state by extrapolating the fit of the Rydberg series to lower lying levels and matching to identified levels [17]. We were able to definitively identify 7 (of the expected 8 ) as-yet unreported $n s$ and $n d$ autoionizing Rydberg series converging to Pd II $4 d^{9}\left({ }^{2} \mathrm{D}_{3 / 2}\right)$. The even-odd mass isotope selective state having the maximum signal from the FC detector is $4 d^{9}\left({ }^{2} \mathrm{D}_{3 / 2}\right) 9 d[3 / 2]_{1}$ at $68,537.93(28) \mathrm{cm}^{-1}$, corresponding to a second laser wavelength of 361.023(3) nm when the intermediate state is $4 d^{9}\left({ }^{2} \mathrm{D}_{3 / 2}\right) 5 p[1 / 2]_{1}$ reached by a $244.865(1)$ $\mathrm{nm}$ [17] first step laser. For this Rydberg state having $J=1$, even-odd mass isotope selectivity is obtained when the relative polarization between first and second lasers is parallel. The selectivity ratio $\frac{\mathrm{Pd}^{\text {odd }}}{\mathrm{Pd}^{\text {all }}}$ was measured to be over $99.7 \%$, as shown in Fig. 5. This high degree of even-odd mass isotope selectivity is obtained when the laser polarization is aligned parallel to the residual magnetic field in the chamber produced by the electron bombardment gun, to minimize magnetic sub-state evolution [20,23]. When the polarizations of both excitation lasers are changed to be perpendicular to the residual magnetic field (maintaining parallel relative polarization), even-odd mass isotope selectivity was observed to degrade to $92.6 \%$.

Table 2 lists the relative signal strengths of the four highest yield Rydberg states, all of which belong to

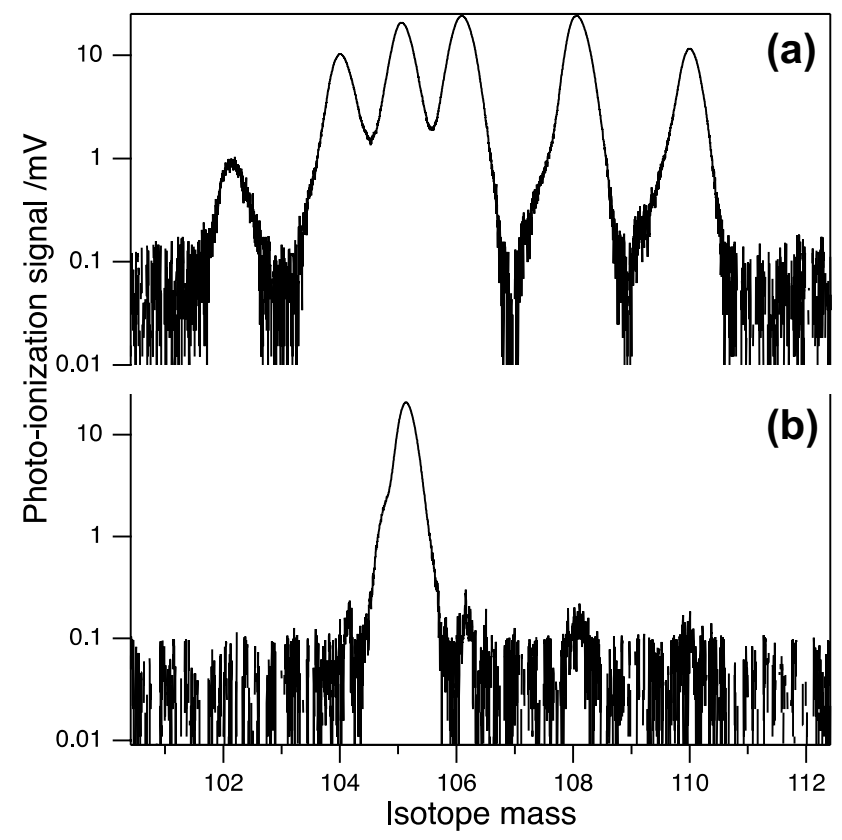

Fig. 5 Photo-ionization signal from Faraday Cup detector showing ionized isotopes using the two-laser scheme at the highest yield Rydberg state of $361.023 \mathrm{~nm}$; a perpendicular polarization between the two excitation lasers, and all isotopes of $\mathrm{Pd}$ are ionized; $\mathbf{b}$ parallel polarization between the two excitation lasers, and only odd-mass number isotopes are ionized. A logarithmic scale is used to display the residual even-mass number isotopes in the selective ionization scheme 
Table 2 Highest efficiency transitions observed using the two-laser scheme

\begin{tabular}{lrl}
\hline Final step wavelength/nm & $n$ & Rel. intensity \\
\hline 371.925 & 8 & 0.23 \\
361.022 & 9 & 1.00 \\
354.288 & 10 & 0.85 \\
349.817 & 11 & 0.81 \\
346.684 & 12 & 0.45 \\
\hline
\end{tabular}

All states are members of Rydberg series $4 d^{9}\left({ }^{2} \mathrm{D}_{3 / 2}\right) n d[3 / 2]_{1}$

$4 d^{9}\left({ }^{2} \mathrm{D}_{3 / 2}\right) n d[3 / 2]_{1}$. A table listing the wavenumber of each peak assigned to the seven Rydberg series is available as supplementary material.

We compare the efficiency of each scheme shown in Fig. 1 by comparing the highest yield states of each and normalizing for laser fluence $\left(\frac{\text { laser pulse energy }}{\text { focal spot area }}\right)$ and number of palladium atoms present in the interaction area as determined from the deposition meter located above the interaction region. For the three-step scheme having ${ }^{2} \mathrm{D}_{5 / 2}$ core intermediate states (scheme A of Fig. 1) the maximum yield is achieved with a final step laser wavelength of $730.89 \mathrm{~nm}$ [11], having an efficiency of 0.02 compared to the two-step excitation scheme (scheme C of Fig. 1). Similarly, for the three-step scheme with ${ }^{2} \mathrm{D}_{3 / 2}$ core intermediate states (scheme B of Fig. 1) the optimal final step laser wavelength is $652.24 \mathrm{~nm}$ [13], resulting in an efficiency 0.13 compared to the two-step excitation scheme, the increase being due to the more efficient auto-ionization via states with ${ }^{2} \mathrm{D}_{3 / 2}$ core (Fig. 6).

\section{Conclusion}

We have demonstrated the viability of the two-laser technique and highlighted its advantages over the conventional three-laser scheme, including: increased yield, reduced cost and complexity, and excellent even-odd mass isotope selectivity. We present definitive identification of seven previously unreported Rydberg series of palladium (four of which are even-odd mass isotope selective), converging to the ionization potential of Pd II $4 d^{9}\left({ }^{2} \mathrm{D}_{3 / 2}\right)$ determined by fits to the extended Ritz formula to be 70,780.67(23) $\mathrm{cm}^{-1}$. The most yield efficient even-odd mass isotope selective Rydberg state is $4 d^{9}\left({ }^{2} \mathrm{D}_{3 / 2}\right) 9 d[3 / 2]_{1}$ at $68,537.93(28) \mathrm{cm}^{-1}$. Identification of the most efficient Rydberg state is an essential and timely step towards the development of large-scale palladium resource recycling and waste reduction projects.

Acknowledgements This work was funded by the ImPACT Program of the Council for Science, Technology and Innovation (Cabinet Office, Government of Japan). This paper is dedicated to RIKEN's centennial.

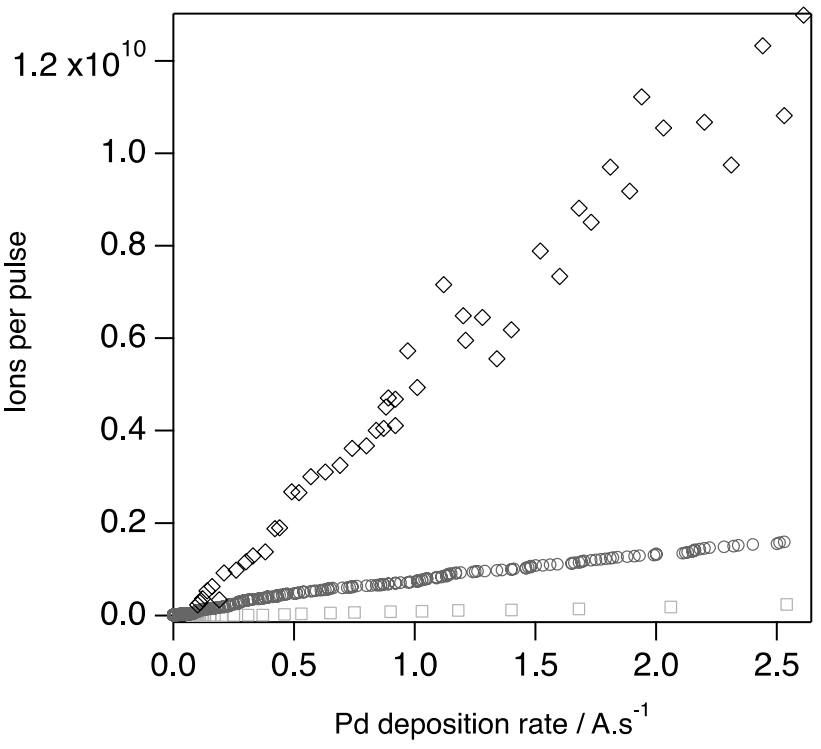

Fig. 6 Observed number of ions per pulse calculated from measurements using the Faraday Cup detector, as a function of Pd evaporation rate measured using a deposition meter located above the interaction region; diamond two-step scheme $\left({ }^{2} \mathrm{D}_{3 / 2}\right.$ core $)$; circle three-laser scheme $\left({ }^{2} \mathrm{D}_{3 / 2}\right.$ core) [13]; square three-laser scheme $\left({ }^{2} \mathrm{D}_{5 / 2}\right.$ core $)[11]$. Laser fluences are normalized to compare the three schemes

Open Access This article is distributed under the terms of the Creative Commons Attribution 4.0 International License (http://creativecommons.org/licenses/by/4.0/), which permits unrestricted use, distribution, and reproduction in any medium, provided you give appropriate credit to the original author(s) and the source, provide a link to the Creative Commons license, and indicate if changes were made.

\section{References}

1. K. Naito, T. Matsui, Y. Tanaka, J. Nucl. Sci. Tech. 23, 540-549 (1986)

2. Impulsing Paradigm Change through Disruptive Technologies (Impact) Program. http://www.jst.go.jp/impact/en/program/08. html. Accessed Aug 2017

3. G. Janes, I. Itzkan, C. Pike, R. Levy, L. Levin, IEEE J. Quantum Electron. 12, 111-120 (1976)

4. E. Van Duijn, S. Witte, R. Zinkstok, W. Hogervorst, Eur. Phys. J. D. 19, 25-29 (2002)

5. L.C. Balling, J.J. Wright, App. Phys. Lett. 29, 411 (1976)

6. H. Yamaguchi, N. Sasao, The Separation of Pd-107 Isotope by Laser, in Proceedings of the International Symposium Advanced Nuclear Energy Research (1989)

7. C.R. Locke, T. Kobayashi, T. Nakajima, K. Midorikawa, Appl. Phys. B 122, 246 (2016)

8. H. Wang et al., Prog. Theor. Exp. Phys. 2017, 021D01 (2017)

9. J. Paisner, Atomic Vapor Laser Isotope Separation. Laser Technology in Chemistry (Springer, Berlin, 1988), pp. 253-260

10. T. Kron, Y. Liu, S. Richter, F. Schneider, K. Wendt, J. Phys. B. 49 185003 (2016)

11. T. Kobayashi, C.R. Locke, K. Midorikawa, Jpn. J. Appl. Phys. 56, 010302 (2016) 
12. R.D. Cowan, The Theory of Atomic Structure and Spectra (Univ of California Press, Berkeley, 1981)

13. C.R. Locke, T. Kobayashi, K. Midorikawa, Appl. Phys. B 123, 33 (2017)

14. M. Baig, A. Rashid, Z. Iqbal, J. Hormes, J. Phys. B. 24, 2295 (1991)

15. N. Karamatskos, M. Müller, M. Schmidt, P. Zimmermann, Phys. Lett. A 102, 409-411 (1984)

16. C. Callender, P.A. Hackett, D.M. Rayner, J. Opt. Soc. Am. B 5, 614-618 (1988)

17. A. Kramida, Y. Ralchenko, J. Reader, NIST ASD Team, Nist Atomic Spectra Database (Version 5.3) (National Institute of Standards and Technology, Gaithersburg, MD, 2015)
18. W. Wiley, I.H. Mclaren, Rev. Sci. Instrum. 26, 1150-1157 (1955)

19. H. Xu et al., Astron. Astrophys. 452, 357-362 (2006)

20. S. Tokita, Y. Izawa, H. Niki, F. Kuwashima, J. Nucl. Sci. Tech. 40, 1014-1018 (2003)

21. W. Ritz, Ann. Phys. 330, 660-696 (1908)

22. D.R. Hartree, The wave mechanics of an atom with a non-Coulomb central field, Math. Proc. Camb. Phil. Soc. 24, 111-132 (1928)

23. H. Niki, I. Kitazima, Y. Izawa, Jpn. J. Appl. Phys. 37, 3343 (1998) 